\title{
Put on a smiley face: Textspeak and personality perceptions
}

Chris Fullwood $^{1 *}$, Sally Quinn ${ }^{2}$, Josephine Chen-Wilson ${ }^{1}$, Darren Chadwick ${ }^{1} \&$ Katie Reynolds $^{1}$

${ }^{1}$ Institute of Psychology, University of Wolverhampton, UK.

${ }^{2}$ Department of Psychology, University of York, UK.

\author{
*Corresponding author \\ Dr Chris Fullwood \\ Institute of Psychology \\ Faculty of Education, Health and Wellbeing \\ University of Wolverhampton \\ West Midlands \\ WV1 1AD \\ UK \\ E-mail: c.fullwood@wlv.ac.uk \\ Phone: +441902323531
}

\begin{abstract}
With the emergence of web 2.0 there has been a dramatic surge in user-generated content. Although the Internet provides greater freedom in self-presentation, computer-mediated communication is characterised by a more relaxed attitude to grammar, spelling and punctuation. The language of the Internet, or textspeak, may be suitable for casual interactions but inappropriate in professional contexts. We tested participant perceptions of an author's personality in two distinct contexts (formal vs. informal) and manipulated the written information under three levels of textspeak: none, low and high. Participants judged the author as less conscientious and less open, but more emotionally stable when textspeak was used, however context had no impact. Personality perceptions of textspeak users differ to those who write in standard English and this is likely to extend to informal impression management contexts (e.g. online dating). These findings also have a number of implications, for example in terms of screening applicants via social media.
\end{abstract}




\section{Introduction}

The Internet is becoming increasingly pervasive and fundamental to daily living. Computermediated communication (CMC) is also impacting globally on the manner in which we meet, interact and relate to others. Alongside the increasing intensity of CMC usage, we are also witnessing arguably the most dramatic transformation of the English language in our history. Furthermore, as a consequence of the extensive use of these technologies, changes spread rapidly and influence vast quantities of people'. Consider the fact that 'selfie' (i.e. a picture taken of oneself by oneself on a smartphone or webcam) was the Oxford Dictionaries' 'word of the year' in $2013^{2}$. Academics also note that some Internet language, which may have initially been expected to remain restricted to text-based communications (e.g. acronyms like 'LOL'), are now being spoken ${ }^{1}$. These are just a couple of examples of how language use is evolving with the advent of CMC. Novel words and phrases are created to describe new forms of online behaviour. Additionally, the structure and composition of language itself is being moulded to meet the interactional needs of online individuals. Although this new form of 'Internet' language has received widespread media coverage and academic attention, few studies have considered its influence on impression formation. This is an important oversight given the prevalence of textspeak use, and the myriad ways in which people gather information and make judgements about others online. This study investigates the impact of varying levels of textspeak on personality perceptions in two communication contexts: an 'about me' section and 'personal statement'.

The label 'textspeak' describes the non-standard, condensed and primarily text-based communication most regularly associated with digital natives in online environments and text-messages ${ }^{3}$. There are numerous idiosyncratic features associated with textspeak, for instance acronyms (e.g. 'brb' for 'be right back'), emoticons (i.e. combinations of keyboard characters denoting facial expressions), shortenings/contractions (e.g. 'txt' for 'text') and non-conventional spellings (e.g. 'dat' for 'that'). Textspeak users can also deliberately omit words, letters and punctuation which they perceive as superfluous ${ }^{3,4}$. There are a number of potential explanations for the popularity of textspeak. For example, it helps users to accommodate for character restrictions (e.g. in text-messages) and increases output speed, so co-communicators do not have to wait too long for a reply. Some forms of textspeak (e.g. emoticons) may also compensate for the lack of non-verbal cues in text-based $\mathrm{CMC}^{3,4}$. 
The ways in which we express ourselves online influence how others perceive us. In various CMC contexts, individuals can make direct identity claims, for example explicitly disclosing interests, hobbies and attitudes. We may also make judgements about others more indirectly through behavioural residue, for example unintentional cues like language choice, and these too provide hints about someone's character ${ }^{5}$. When it comes to managing impressions, the Internet can be a double-edged sword. On the one hand, we have increased flexibility in selfpresentation. In asynchronous communication modes, we can more carefully consider message content so we come across more desirably, for example by re-reading and editing messages before sending. This could potentially lead to an 'optimal' presentation of the self, leading to more favourable impressions ${ }^{6,7}$. On the other hand, CMC is typically more informal, loosely-structured, and not bound by the same conventions of grammar, spelling and punctuation as other forms of written communication might $\mathrm{be}^{8,9}$. Therefore in CMC contexts, for example social networking sites (SNS), there will be increased instances of slang, colloquialisms and textspeak ${ }^{8,10}$.

Communicating in textspeak, as opposed to standard English, is likely to lead to different types of impressions, and this typically depends on the communication context. Although textspeak may be deemed 'appropriate' in informal contexts (e.g. texting friends), it may be considered unsuitable in professional communications (e.g. a résumé) ${ }^{10}$. Textspeak users may even receive less positive evaluations in more informal, impression-management contexts, particularly if co-communicators are making judgments about the individual based on what they write. For example, Ellison et al. ${ }^{11}$ noted that stylistic features (e.g. grammar) of online dating profiles were as influential as the actual content in guiding fellow daters' judgements. Many participants found poor grammar (which is associated with textspeak) off-putting. Organisations and employers are also increasingly screening candidates and applicants via SNS to gather additional information which may not be communicated in a résumé or personal statement ${ }^{12,13}$. Although this strategy may aid the employer to discover additional clues about the applicant's character, textspeak users may be disadvantaged if negative impressions are formed on the basis of their online language use.

A recent study by Scott and colleagues ${ }^{10}$ demonstrates that individual impressions vary with textspeak use. In their experiment, six Facebook profiles were created which varied in terms of the language used in the profile owner's status updates. Some profiles contained no 
spelling or grammatical errors, others contained such errors and some were written partly in textspeak. Profile owners using correct language were deemed more intelligent, competent and employable than those using textspeak or incorrect language, however attractiveness ratings were equivalent. Although using textspeak does not necessarily suggest poorer literacy ability - indeed, many studies actually suggest that textspeak users have better literacy $^{14,15}$ - impressions associated with such users were more negative nonetheless. Thus, even though employing textspeak in CMC may be a conscious strategy (and not necessarily because one cannot spell particular words/phrases), it appears that users are judged as though they have made language errors ${ }^{10}$. Although certain elements of textspeak (e.g. non-standard spellings) may lead to negative perceptions, this is not to say that all textspeak types should necessarily be considered as the same. Indeed, there is some evidence that emoticon users are perceived as more agreeable and extravert than non-users in informal instant-messenger communications $^{16}$, perhaps because emoticons allow greater insight into the user's disposition and help compensate for emotional cue-restriction.

The primary aim of this research was to increase knowledge of how textspeak use influences impression formation. Although previous research suggests that certain elements of textspeak may detrimentally affect impressions ${ }^{10,11}$, no study to date has compared this across different contexts. For example, we know that textspeak use in SNS is relatively commonplace, but it is also apparent that employers are increasingly noting the use of textspeak in résumés, cover letters and job applications by young people ${ }^{17,18}$ and $64 \%$ of teens report to have used textspeak in their academic writing ${ }^{19}$. Some contexts may be deemed suitable to use textspeak, whereas others may not. This study will therefore compare impressions across two distinct contexts: an 'about me' section of an SNS profile, and a 'personal statement' attached to a University application.

Additionally, no research has considered whether the volume of textspeak influences impressions. Therefore, three levels were assessed: standard English (no grammatical or spelling errors), low textspeak and high textspeak. Impressions were measured in terms of personality perceptions, specifically self-esteem and the Big Five traits. As well as providing a relative judgement about whether textspeak users are perceived more negatively than nonusers, gathering personality perceptions should also shed some light on the different traits attributed to textspeak users. Although there is a lack of previous research to guide specific predictions regarding each personality dimension, it was expected that the standard English 
condition would lead to more positive assessments. As we expected the 'about me' section to be a more appropriate context in which to use textspeak, we also predicted more negative perceptions of the textspeak user in the 'personal statement' condition. Finally, it was anticipated that perceptions would be more negative as textspeak volume increased.

\section{Method}

\section{Participants}

In the study 167 volunteers (62 males; 105 females) participated. The mean age was 26.41 years (standard deviation 10.52), ranging from 16 to 61 years. Participants were recruited in various ways: via the Psychology department's participant pool, by advertising the study on a number of relevant forums, and via word of mouth. The final sample comprised 121 students (72\%) and 46 non-students. Participants were exposed to one of six variations of text content. There were no significant differences between the six variations in terms of $\operatorname{sex}\left(X^{2}(\mathrm{df}=5)\right.$ $=5.34 ; p=0.376)$ and age $\left(F^{5,161}=0.279, p=0.924\right)$.

\section{$\underline{\text { Materials }}$}

Three 'about me' sections and 3 'personal statements' were created, and the information conveyed was identical in each. To ensure the content was reasonably representative of both a typical 'about me' and 'personal statement' a pilot study was conducted on a separate group of 20 volunteers: 10 rated the standard English 'personal statement' and 10 rated the equivalent 'about me' section. Participants were asked: 'how typical is this personal statement/about me section in terms of the content, style and length?' An independent measures t-test revealed no significant difference, with each condition scoring a mean of 2.70 out of a possible 5. Therefore the content was considered to be averagely representative of the two contexts and there was no difference in the perceived typicality of the two conditions. The 3 versions within each communication context differed in terms of textspeak volume (standard English, low textspeak and high textspeak). The sex of the author was not made explicit in the text. The low and high textspeak texts were created by substituting words and phrases in the standard English text with textspeak. Textspeak examples included acronyms (e.g. 'atm'), g-clippings (e.g. 'readin'), non-conventional spellings (e.g. 'dat'), contractions (e.g. 'hrs') and emoticons. Each unit of text was approximately 380 words. The proportion of textspeak in the low and high textspeak conditions was $8 \%$ and $46 \%$, respectively. 
Big Five personality perceptions were measured using the 50-item International Personality Item Pool $^{20}$. The Rosenberg self-esteem scale ${ }^{21}$ was used to measure perceptions of the author's self-esteem. For each questionnaire instructions and questions were rephrased asking participants to rate the text author, rather than themselves. A number of previous studies have utilised a similar approach to measuring personality perceptions of others (e.g. Markey \& Wells ${ }^{22}$; Fullwood \& Martino ${ }^{16}$ ) therefore supporting the design of this study.

\section{Procedure}

Participants were directed to an online survey. The information page outlined their role within the study and informed participants that the text they were about to see had been taken from a real 'about me' or 'personal statement', but the name had been removed to protect the individual's identity. Consent to participate was given by clicking a button onscreen. Participants were randomly exposed to one of the 6 variations. After reading the text, they were then requested to fill out two questionnaires to assess their perceptions of the author. Once completed, debrief information was presented where participants were informed about the deception - i.e. that the text was created by the researchers. Participants were given the opportunity at this point to withdraw their data from the study. Researchers' contact details were also supplied if participants required further information.

\section{Results}

Ratings on each of the Big Five traits and self-esteem were entered into a 2 x 3, betweensubjects ANOVA, with Communication Context ('about me' and 'personal statement') and Textspeak Level (standard English, low textspeak and high textspeak) as the factors. Means and standard deviations are in Table 1.

Table 1: Means and standard deviations (in brackets) for participant perceptions of the text author's self-esteem and Big Five traits for each of the three textspeak levels.

\begin{tabular}{|l|l|l|l|l|l|l|}
\hline $\begin{array}{l}\text { Text } \\
\text { Level }\end{array}$ & Self-esteem & $\begin{array}{l}\text { Extra- } \\
\text { version }\end{array}$ & $\begin{array}{l}\text { Agree- } \\
\text { ableness }\end{array}$ & $\begin{array}{l}\text { Conscient- } \\
\text { iousness }\end{array}$ & $\begin{array}{l}\text { Emotional } \\
\text { Stability }\end{array}$ & Openness \\
\hline None & $22.67(5.32)$ & $29.87(3.73)$ & $31.29(4.03)$ & $31.15(3.85)$ & $27.09(6.41)$ & $32.23(4.28)$ \\
\hline Low & $21.19(4.11)$ & $28.84(3.23)$ & $29.87(3.72)$ & $28.35(3.85)$ & $29.58(3.62)$ & $28.44(4.71)$ \\
\hline High & $19.61(3.79)$ & $29.22(2.47)$ & $30.55(3.52)$ & $27.41(4.89)$ & $30.08(5.18)$ & $28.33(4.27)$ \\
\hline
\end{tabular}


For self-esteem there was a significant main effect of Textspeak Level $\left(F^{2,161}=6.08, p<0.01\right.$, the effect size (partial Eta squared) was $=0.070$ ). Post hoc tests (Games-Howell) revealed a significant difference between 'standard English' and 'high textspeak' $(p<0.01)$. The author was deemed to possess significantly higher self-esteem in the 'standard English' condition compared to the 'high textspeak' condition.

For conscientiousness, there was a significant main effect of Textspeak Level $\left(F^{2,161}=12.29\right.$, $p<0.01$, partial Eta squared $=0.133$ ). Significant differences between 'standard English' and 'high textspeak' $(p<0.01)$ and between 'standard English' and 'low textspeak' $(p<0.01)$ were found. Textspeak use (irrespective of the amount) resulted in lower ratings of conscientiousness than text written in standard English.

For openness, there was a significant main effect of Textspeak Level $\left(F^{2,161}=15.39, p<0.01\right.$, partial Eta squared $=0.160$ ). Significant differences between 'standard English' and 'high textspeak' $(p<0.01)$ and between 'standard English' and 'low textspeak' $(p<0.01)$ were found. Textspeak use resulted in lower ratings of openness compared to text written in standard English.

For emotional stability, there was a significant main effect of Textspeak Level $\left(F^{2,161}=5.29\right.$, $p<0.01$, partial Eta squared $=0.062$ ). Significant differences between 'standard English' and 'high textspeak' $(p<0.01)$ and between 'standard English' and 'low textspeak' $(p<0.05)$ were revealed. Textspeak use resulted in higher ratings of emotional stability than text written in standard English.

There were no significant main effects for Communication Context and no significant interactions (Context x Textspeak Level) for any of the DVs.

Additionally, a series of independent samples t-tests revealed no significant differences on any of the dependent variables between the sexes. Furthermore, Spearman's correlations show no significant relationships between age and any of the dependent variables.

\section{Discussion}

A significant advantage of the methodology employed was that the information in each variation was identical. Therefore, we can be more confident that any differences in 
participant perceptions are a consequence of the level of textspeak. The qualities and characteristics we associate with text-speakers appear to be quite distinct from those we associate with individuals who write formally, where their competence or attitude to work was concerned. The author was judged as less conscientious and less open when textspeak was used, irrespective of the amount. In this sense, textspeak has a detrimental impact on perceptions of an unfamiliar person, mirroring the negative impressions reported by Scott et $\mathrm{al}^{10}$. On the other hand, the perception of the author's more 'personable' or 'social' quality was not affected by the presence of textspeak in the text. No significant main effect of textspeak was found in the rating of the author's agreeableness and extraversion.

Somewhat surprisingly, findings also demonstrate that the amount of textspeak used is not particularly important in terms of influencing perceptions. Even a small amount of textspeak was sufficient to alter perceptions. However, only the author in the 'high' textspeak condition was deemed to have poorer self-esteem than the perfect English condition. So, a particularly high level of textspeak seems necessary to sway the opinions of others that the individual has a lower level of self-worth. Participants may have considered abundant textspeak to reflect a lack of intelligence, and that the user was unsatisfied with him/herself for this reason.

Although textspeak altered perceptions of an individual to some extent, contrary to expectations there were no differences between the communication contexts and no interactions between the two main effects. We expected ratings of the textspeak-using author to be more negative in the 'personal statement' because this context would be deemed unsuitable to write in textspeak. The fact that there were no differences may say something about the purpose of an 'about me' section. Indeed, Fullwood et al. ${ }^{23}$ describe the 'about me' as a 'social CV.' In essence, this is where the profile owner sells him/herself socially to others. The information contained within this section may be used to appraise the individual and make decisions about whether or not he or she should be added to others' networks. It could be argued that, although in very different ways, the 'about me' section and 'personal statement' are both impression management contexts. Further research should therefore assess the impressions formed of textspeak users in a variety of CMC contexts, including ones which are not directly concerned with impression management. Alternatively, the general education level of the sample may have had an influence on the findings. University students will encounter a variety of situations in which they are required to write very 
formally. This may have led to a negative bias towards informal writing, irrespective of the context in which it is presented.

Finally, textspeak use increased ratings of emotional stability. In explaining this finding, it may be suggested that the addition of emoticons, and other markers of emotion, helped to close the intimacy distance between the author and rater in cyberspace. Indeed, Liu et al. ${ }^{24}$ note that people experience increased intimacy when communicating with textspeak as they are more likely to feel that they are in an exchange with a real person. The increased emotional tone as well as more individualised communications associated with such uses of textspeak may partly explain this particular finding. Further, this also implies that perceptions of textspeak users are not always necessarily negative and that not all examples of textspeak are the same. For example, emoticons have been shown to improve ratings of authors ${ }^{16}$. Different types of textspeak may therefore influence different attributions of the author. Further research should explore this idea in more detail, for example by comparing the perceptions of individuals who exclusively utilise individual textspeak types.

One potential limitation of this study should be addressed in future research. The sample consisted of mainly females (63\%) and chiefly comprised students (72\%). Further research may therefore explore whether the types of personality perceptions found in this study also hold true in a sample consisting of individuals from more varied backgrounds (particularly in terms of education level). It would also be particularly interesting to note if the same effects can be found in a sample of participants who are involved in making hiring decisions on a regular basis (e.g. admissions officials).

In conclusion, textspeak authors were perceived to be less conscientious, less open and to have lower levels of self-esteem than their counterparts with a formal writing style. However, textspeak authors were also perceived to have greater emotional stability than standard English authors. It is suggested that some forms of textspeak may improve ratings while other forms might signal a lack of attention to detail or an inability to write coherently and intelligently. Hence, people who choose to communicate in this way indiscriminately may be well served by giving greater consideration to how such use might affect others' perceptions of them and their career prospects. Moreover, further research should address the usefulness of screening applicants based on their online communication styles as organisations might not be aware of these biases when making initial judgements of unfamiliar persons. Without 
further evidence about the accuracy of such judgements, the presence of textspeak may lead to stereotypes rather than accurate assessments of an individual.

\section{Author Disclosure Statement}

No competing financial interests exist.

\section{References}

1. Kinsella N. Btw its just netspeak lol. Griffith Working Papers in Pragmatics and Intercultural Communication 2010; 3(2): 64-74.

2. The Guardian. (Tuesday 19 November, 2013). Selfie is Oxford Dictionaries' word of the year. Retrieved on 10 July, 2014 from http://www.theguardian.com/books/2013/nov/19/selfie-word-of-the-year-oedolinguito-twerk

3. Farina F, Lyddy F. The language of text messaging: "Linguistic ruin" or resource? The Irish Psychologist 2011; 37(6): 145-149.

4. Crystal D. (2008) Txting: The Gr8 Db8. Open University Press: Oxford.

5. Gosling SD, Ko SJ, Mannarelli T, Morris ME. A room with a cue: Personality judgments based on offices and bedrooms. Journal of Personality and Social Psychology 2002; 83(3): 379-398.

6. Walther JB. Computer-mediated communication: impersonal, interpersonal, and hyperpersonal interaction. Communication Research 1996; 23: 3-43.

7. Walther JB, Parks M. (2002) Cues filtered out, cues filtered in. In: Knapp ML, Daly JA (Eds) Handbook of interpersonal communication 2002 (pp. 529-563). Thousand Oaks, CA: Sage.

8. Drouin MA. College students' text messaging, use of textese and literacy skills. Journal of Computer Assisted Learning 2011; 27: 67-75.

9. Crystal D. (2006) The fight for English: How language pundits ate, shot and left. Oxford University Press: Oxford.

10. Scott GG, Sinclair J, Short E, Bruce G. It's not what you say, it's how you say it: Language use on Facebook impacts employability but not attractiveness.

Cyberpsychology, Behavior and Social Networking in press.

11. Ellison N, Heino R, Gibbs J. Managing impressions online: Self-presentation processes in the online dating environment. Journal of Computer-Mediated Communication 2006; 11(2): 415-441.

12. Slovensky R, Ross WH. Should human resource managers use social media to screen job applicants? Managerial and legal issues in the U.S.A. Info 2012; 14(1): 55-69.

13. Davison HK, Maraist CC, Hamilton RH, Bing MN. To screen or not to screen? Using the Internet for selection decisions. Employee Responsibilities and Rights Journal 2012; 24: 1-21.

14. Kemp N, Bushnell C. Children's text messaging: abbreviations, input methods and links with literacy. Journal of Computer Assisted Learning 2011; 27: 18-27.

15. Plester B, Wood C, Joshi P. Exploring the relationship between children's knowledge of text message abbreviations and school literacy outcomes. British Journal of Developmental Psychology 2009; 27: 145-161. 
16. Fullwood C, Martino OI. Emoticons and impression formation. Applied Semiotics 2007; 19: 4-14.

17. Kotzee B, Johnston R. 'Can't string a sentence together?' UK employers' views of graduates' writing skills. Industry and High Education 2011; 25(1): 45-52.

18. Employers are from Mars, young people are from Venus: Addressing the young people/jobs mismatch. Chartered Institute of Personnel and Development (CIPD) in association with Business in the Community (BITC), April 2013. Retrieved on 10 July, 2014 from http://www.cipd.co.uk/binaries/MarsVenus\%20FINAL\%2030\%2004\%2013.pdf

19. Lenhart A, Arafeh S, Smith A, Macgill AR. Writing, Technology and Teens. Pew Internet and American Life Project report 2008. Retrieved on 10 July, 2014 from http://pewinternet.org/

20. Goldberg LR. A broad-bandwidth, public domain, personality inventory measuring the lower-level facets of several five-factor models. In: Mervielde I, Deary I, De Fruyt F, et al. (eds) Personality Psychology in Europe, vol. 7 1999. Tilburg: Tilburg University Press, pp. 7-28.

21. Rosenberg M. (1965) Society and the adolescent self-image. Princeton, NJ: Princeton University Press.

22. Markey PM, Wells SM. Interpersonal perception in Internet chat rooms. Journal of Research in Personality 2002; 36: 134-146.

23. Fullwood C, Evans L, Morris N. Linguistic androgyny on MySpace. Journal of Language and Social Psychology 2011; 30(1): 114-124.

24. Liu YC, Lin C, Huang YA. An exploration of communicating with textspeak: A social presence perspective. The International Technology Management Review 2012; 3(1): 54-59. 
Table legend

Table 1: Means and standard deviations (in brackets) for participant perceptions of the text author's self-esteem and Big Five traits for each of the three textspeak levels. 